DOI: https://dx.doi.org/10.26808/rs.ca.i8v1.24

\title{
Analysis of Low Level Energy Emissions
}

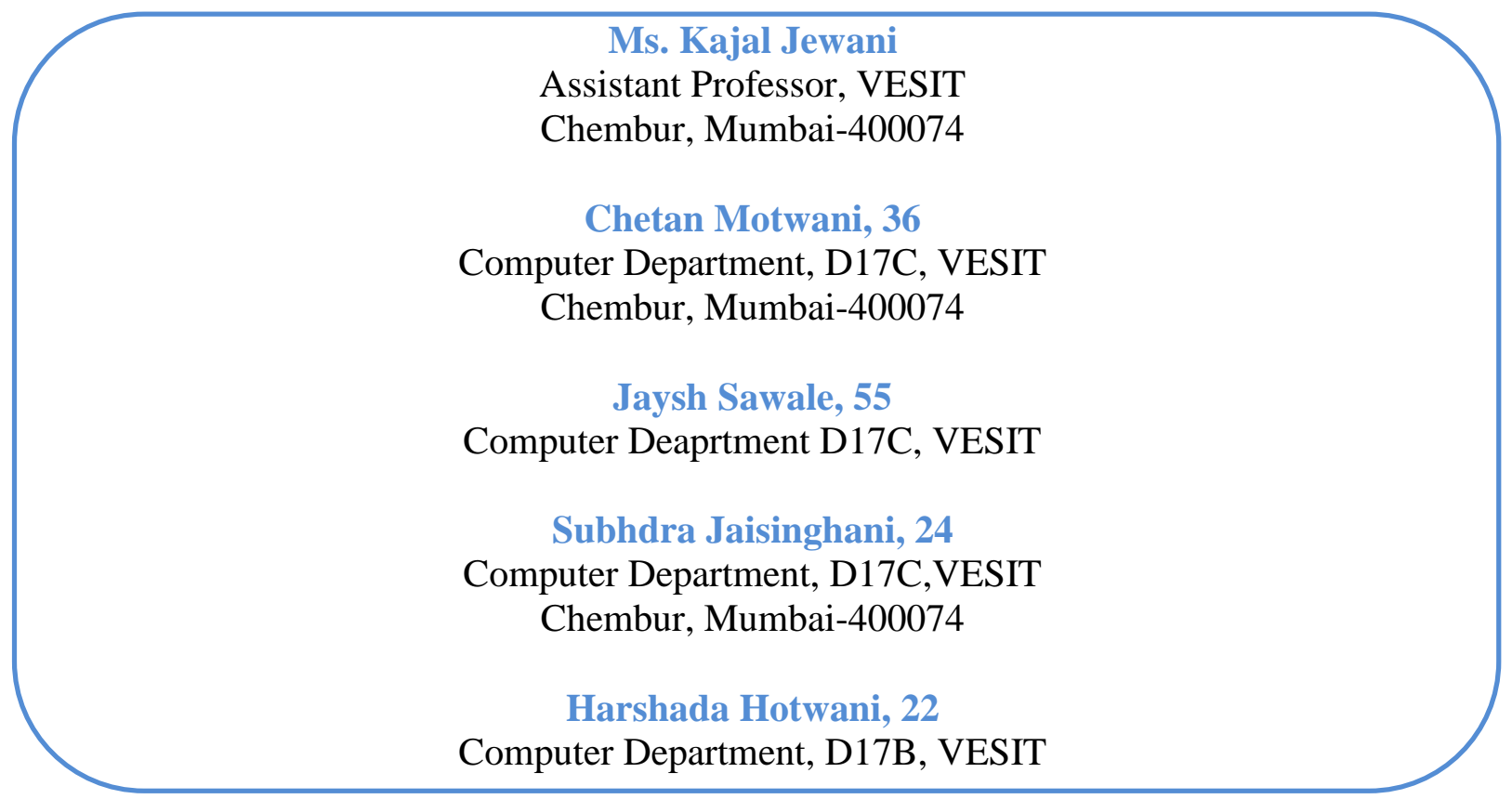

\section{Abstract}

In this paper, we have discussed about various techniques of how to capture the low-level energy which is being emitted from the objects, and to study the variations in energy of different things, and also to process the image captured and will produce the final output, from which we can extract the information to analyze the digital light state of object. All objects and living organisms, including humans, emit a low-level intensity glow that cannot be seen by naked eye. This low-level intensity glow is also called as Biophoton emission or Bioluminescence. We aim at capturing these bio-photons emitted from an object.

Keywords - Biophotons, Bioluminescence, CCD, GDV, RFI

\section{INTRODUCTION}

Light is not only what brightens up our whole day,makes us see the things around us, but it is also produced by our own cells and forms a major component of man's inner environment and nonmaterial part of our body connecting us with the external environment.

All objects and living organisms, including humans, emit a low level intensity glow that cannot be seen by naked eye.

This low level intensity glow is also called as Bio-photon emission or Bio-luminescence.

Bio-photons are photons of light in the ultraviolet and low visible light range that are produced by a biological system. They are non-thermal in origin, and the emission of bio-photons is technically a type of bio-luminescence, though bio-luminescence is generally reserved for higher luminance lucifer in/luciferase systems. The term bio-photon used in this narrow sense should not be confused with the broader field of bio-photonics, which studies the general interaction of light with biological systems. Biological tissues typically produce an observed radiant emittance in the visible 
DOI: https://dx.doi.org/10.26808/rs.ca.i8v1.24 International Journal of Computer Application (2250-1797)

Issue 8 Volume 1, January- February 2018

and ultraviolet frequencies ranging from $10^{-17}$ to $10^{-23} \mathrm{~W} / \mathrm{cm}^{2}$ (approx $1-1000$ photons $/ \mathrm{cm}^{2} / \mathrm{second)}{ }^{[1]}$ This low level of light has a much weaker intensity than the visible light produced by bio-luminescence, but bio-photons are detectable above the background of thermal radiation that is emitted by tissues at their normal temperature.

\section{METHODS}

1. Bio-photon Method.

Bio-luminescence, which is weak but visible, is some times produced in living organisms, such as fireflies or jellyfish, as the result of specialized enzymatic reactions that require adenosine triphosphate. However, all living organisms emit extremely weak light, spontaneously without external photo excitation. This biophoton emission is categorized in different phenomena of light emission from bioluminescence, and is believed to be a by-product of biochemical reactions in which excited molecules are produced from bioenergetic processes that involves active oxygen species . Human body is glimmering with light of intensity weaker than 1/1000 times the sensitivity of naked eyes . By using a sensitive charge-coupled-device (CCD) camera with the ability to detect light at the level of a single photon, we succeeded in imaging the spontaneous photon emission from human bodies .. Previously, for obtaining an image, it took more than 1 hour of acquisition, which is practically impossible for the analysis of physiologically relevant bio-photon emission. By improving the CCD camera and lens system, here we have succeeded in obtaining clear images using a short exposure time, comparable with the analysis of physiological phenomena. Since metabolic rates are known to change in a circadian fashion, we investigated the temporal variations of biophoton emission across the day from healthy human body.

Also ased on the photoelectric effect, appropriate photomultiplier systems have been developed in order to detect this very weak light. Although Biophotons emission correlates strongly with all the life activities of organism. Biophysical research has measured low light impulses,called biophotonic emission, in cells and biological tissue It is now known that all cells (plant, animal or human) emit a weak, called biophotonic radiation.

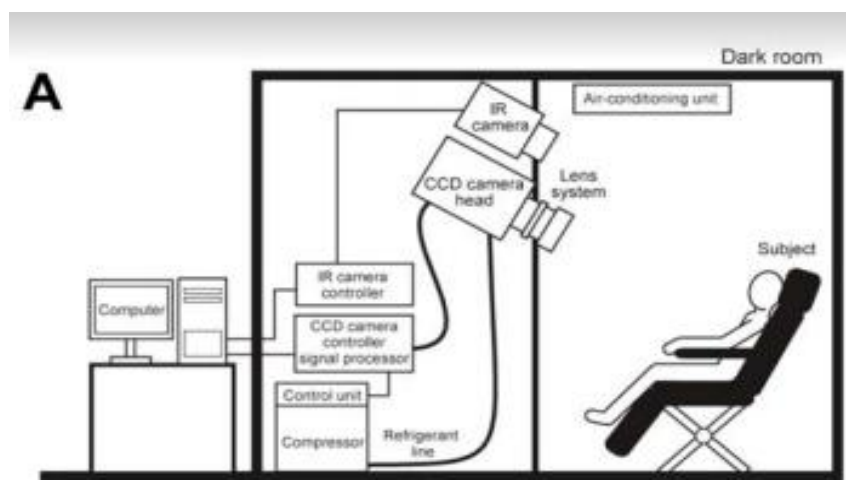

Fig. 1a: Block Diagram of CCD camera

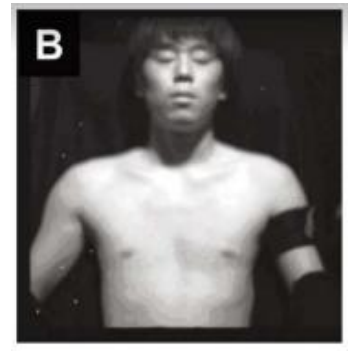

Fig. 1b: Input Image

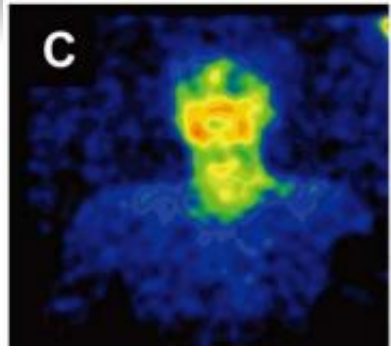

Fig. 1c: Output Image 
DOI: https://dx.doi.org/10.26808/rs.ca.i8v1.24 International Journal of Computer Application (2250-1797) Issue 8 Volume 1, January- February 2018

\section{Gas Discharge Visualization (GDV)}

Throughout human history, there has been a belief that all humans have a bio-energy or aura which surrounds their entire body. With the advancement in science and technology, the phenomenon of bio-energy has been concluded to be electromagnetic field (EMI) generated by the body itself. When applied on the diagnostics of human health, the common term used to describe this energy is Bio-energy; energy emitted by biological system. The bio-energy presented in this paper is based on coronal discharge images of the fingers captured through BEO GDV (Biological Emission and Optical radiation, stimulated by electromagnetic field, amplified by Gas Discharge with Visualization through computer data processing) developed by Konstantin Korotkov . The technique is based on the occurrence of coronal discharge when a high intensity electric field is created around an object as its basic fundamental principle. Since photon emission accompanies such phenomenon, it is therefore possible to capture and analyze this coronal discharge as a photographic image through appropriate software. Combining with the fundamentals of Traditional Chinese Medicine (TCM) in meridian channels, the system is able to provide an overall health status of a patient. This is achieved by capturing and analyzing the images from all ten fingers which lies on the meridians thus providing feedbacks of the major systems and organs, which they are linked to. The bio-electromagnetic field or aura mention in this study can be explained as the result of induction within the neural and meridian pathways in the body, as an electrical impulse passing through them. The phenomenon causes the ionization of molecules within the gases around the skin. With the fingers relatively positively charged, negatively charged electrons projected from the dielectric plate will be absorbed by the fingers as the plate within the GDV equipment is energized (Figure 1a and 1b).

As electrons move through the air, collision between the electrons and the gas molecules wrenches out more electrons and induces ionization of the gas molecules. Thus, the light emission resulting from the impact, generates branching tree-like patterns around each of the fingers.

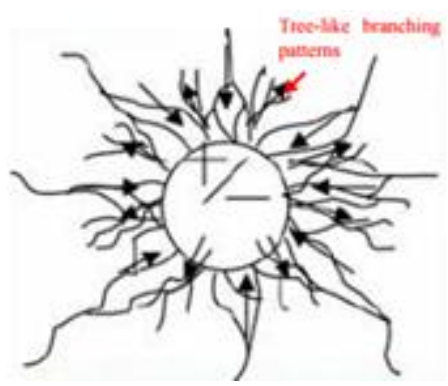

Fig. 2a: Impact of alternating current upon gas discharge.

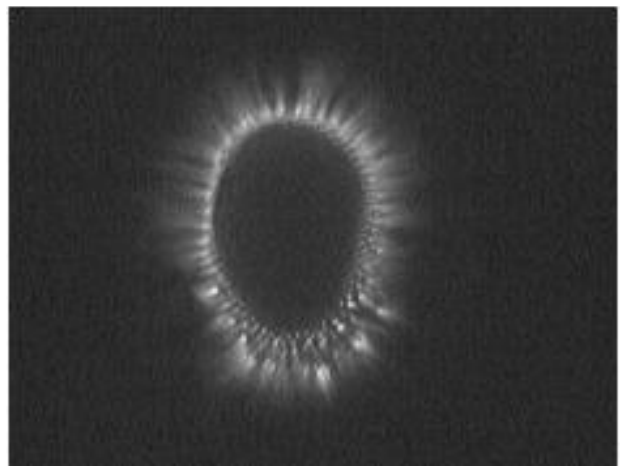

Fig. 2b: Actual image of finger. 


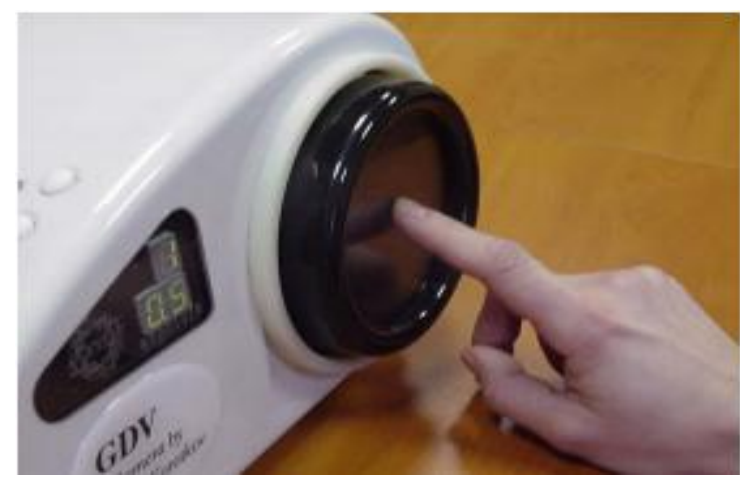

Fig. 2c: Capturing of an image with the camera.

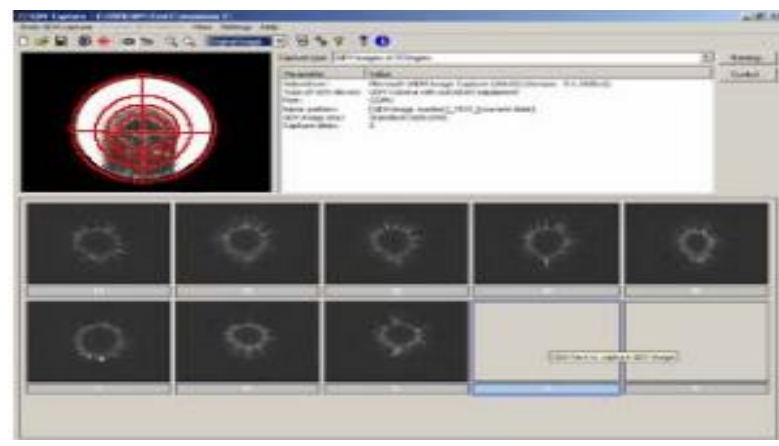

Fig. 2d: Processinf of images through BEO GDV system.

\section{Resonant Frequency Imaging (RFI)}

Resonant Field Imaging (RFI) is a type of Magnetic Resonant Imaging (MRI). RFI is an electromagnetic feedback and imaging process. This technology provides detailed scientific information and objective interpretations for all auras, low level energies emitted from bio-logical tissues and identifies the type and function of all bio-energies present in specific regions of human brain. The RFI system accurately identifies and interprets 15 colors of bio-energy, representing all 15 distinguishable colors of the optical spectrum, giving it the maximum possible usefulness for detailed and accurate images and interpretations. The RFI system employs a hand-held frequency meter with a specially tuned antenna that measures the frequency in megahertz around the body at various points and distances. It gives a true, real-time reading of the aura frequencies in the natural state at the point of testing. Each frequency can be correlated to one of the fifteen colors on a frequency chart to assist in looking at the aura colors. The computer software program that accompanies the RFI system takes the entered frequencies, prints the color chart of the aura colors of health level and then prints a complete printout of the meaning of color in the specific areas.

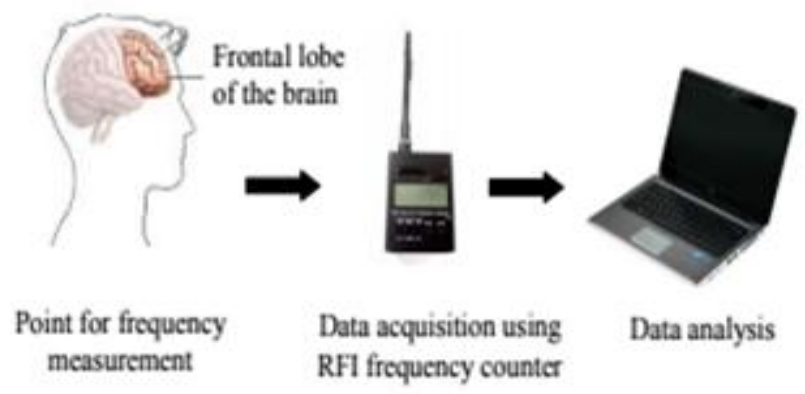

Fig. 3a: The setup for the measurement of bioenergy frequency. 


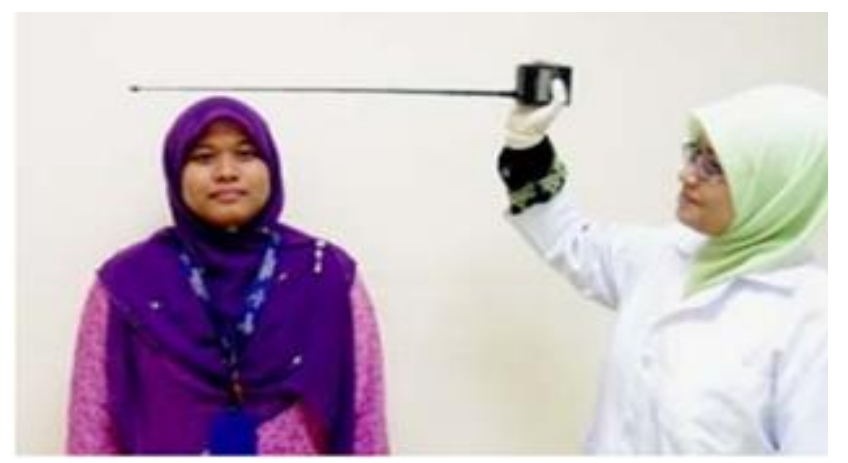

Fig. 3b: The measurement of bioenergy frequency.

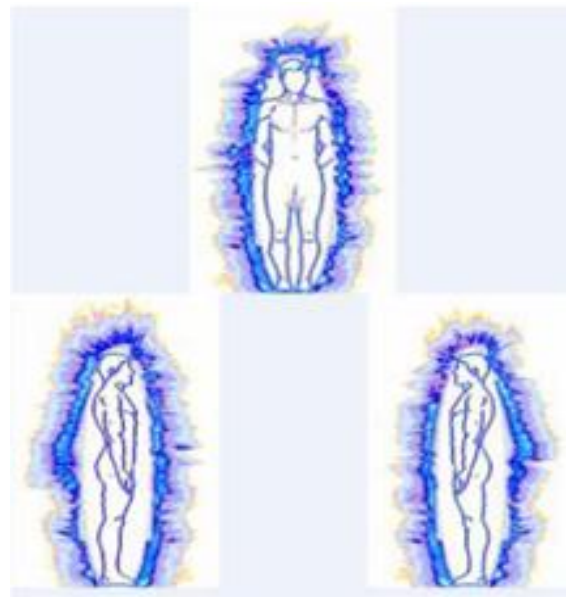

Fig. 3c: Result of a male stroke patient.

\section{RESULTS}

After studying various methods of detecting bio-photons, it is clear that bio-photons are really emitted from all the objects and the intensity of this emission vary day after day. Also these biophoton can communicate, as they are stored in human's cell and DNA.

\section{CONCLUSION}

The techniques described in this paper are the best techniques, but they are available at few organizations are they are expensive. Outputs obtained from these methods are satisfactory and provides a detailed information about human's living system and their culture. This information can be used for the further analysis by the practitioners and healers for improving the well being of the humans.

\section{REFERENCES}

[1] Imaging of Ultraweak Spontaneous Photon Emission From Human Body Displaying Diurnal Rhythm- July 2009 | Volume 4 | Issue 7 | e6256.

[2] Bioenergy based Medical Diagnostic Application based on Gas DischargeVisualization- 07803-8740-6/05/\$20.00 @2005 IEEE.

[3] Bioenergy Frequency Measurement of Stressed and Non-stressed Individuals Using Resonant Field Imaging (RFI) Frequency Counter- 978-1-47994084-4/14/\$31.00 @2014 IEEE 